\title{
Knowledge, Attitudes and Practices of Populations Towards Barrier and Preventive Measures Against COVID-19 in Two Cities in Cameroon
}

\author{
Cécile Ingrid Djuikoue $^{1,2}$, Elodie Ngazoue ${ }^{3,4}$, Gildas Tazemda ${ }^{1}$, Rodrigue Kamga Wouambo ${ }^{2,4}$, \\ Agbor Michael Ashu ${ }^{1}$, Mariella Leumi ${ }^{1}$, Esemu Livo ${ }^{5}$, Héritier Malengele Mawalala ${ }^{6}$, \\ Yvonne Valérie Yolande Voumbo Matoumona Mavoungou ${ }^{7}$, Aliocha Natuhoyila Nkodila ${ }^{6}$, \\ Eugène Ndebia $^{8}$, Nombulelo Chitha ${ }^{9}$, Wezile Chita ${ }^{9}$, John Sungwacha Nasila ${ }^{9}$, \\ Omer Tchikamgoua Njajou ${ }^{2}$, Venant Tchokonte-Nana ${ }^{1,}$, , Benjamin Longo-Mbenza ${ }^{6,8}$, \\ Charles Awono Onana ${ }^{1}$
}

${ }^{1}$ Faculty of Health Sciences, University des Montagnes, Bangangte, Cameroon

${ }^{2}$ Prevention and Control Foundation, Yaounde, Cameroon

${ }^{3}$ Department of Microbiology, Estuary Academy and Strategic Institute (IUEs/ INSAM), Yaounde, Cameroon

${ }^{4}$ Department of Microbiology and Parasitology, Faculty of Science, University of Buea, Buea, Cameroon

${ }^{5}$ Center for Research in Emerging and Reemerging Diseases, Institute for Medical Research and Medical Plants Studies, yaounde, Cameroon ${ }^{6}$ Faculty of Public Health, LOMO University of Reseach, Kinshasa, Democratic Republic of Congo

${ }^{7}$ Faculty of Health Sciences, University Marien Ngouabi, Brazzaville, Republic of Congo

${ }^{8}$ Faculty of Health Sciences Walter Sisulu University, Eastern Cape, Mthatha, South of Africa

${ }^{9}$ Health Systems Enablement \& Innovation Unit, University of the Witwaterstand, Johannesburg, South of Africa

\section{Email address:}

venant.tnana@gmail.com (V. Tchokonte-Nana), Djuikoe1983@yahoo.fr (C. I. Djuikoue), florngazoue@gmail.com (E. Ngazoue), drtazemda@gmail.com (G. Tazemda),rodriguekamga89@yahoo.fr (R. K. Wouambo), agborasm@gmail.com (A. M. Ashu), leumimariella@gmail.com (M. Leumi), esemu_livo@yahoo.com (E. Livo), herimawalala@gmail.com (H. M. Mawalala), yvoumbo@yahoo.fr (Y. V. Y. V. M. Mavoungou), nkodilaaliocha@gmail.com (A. N. Nkodila), ndebiaeugene@gmail.com (E. Ndebia), nchita@wits.co.za (N. Chitha), wezile.chitha@wits.co.za (W. Chita), nasila.john@gmail.com (J. S. Nasila), njajou@gmail.com (O. T. Njajou), longombenza@gmail.com (B. Longo-Mbenza), awonoonana.charles@gmail.com (C. A. Onana) ${ }^{*}$ Corresponding author

\section{To cite this article:}

Cécile Ingrid Djuikoue, Elodie Ngazoue, Gildas Tazemda, Rodrigue Kamga Wouambo, Agbor Michael Ashu, Mariella Leumi, Esemu Livo, Héritier Malengele Mawalala, Yvonne Valérie Yolande Voumbo Matoumona Mavoungou, Aliocha Natuhoyila Nkodila, Eugène Ndebia, Nombulelo Chitha, Wezile Chita, John Sungwacha Nasila, Omer Tchikamgoua Njajou, Venant Tchokonte-Nana, Benjamin Longo-Mbenza, Charles Awono Onana. Knowledge, Attitudes and Practices of Populations Towards Barrier and Preventive Measures Against COVID-19 in Two Cities in Cameroon. World Journal of Public Health. Vol. 6, No. 3, 2021, pp. 89-98. doi: 10.11648/j.wjph.20210603.13

Received: May 25, 2021; Accepted: June 7, 2021; Published: August 5, 2021

\begin{abstract}
Coronavirus Disease 2019 (COVID-19) is a contagious disease caused by the severe acute respiratory syndrome coronavirus 2 (SARS-CoV-2). Since the first reported case in sub Saharan Africa, countries have struggled to respond to the pandemic despite all the barrier measures and preventions strategies put in place; amongst them, Cameroon is characterized by a large amount of contamination and an increase in death rates. The purpose of this study was to assess the knowledge, attitudes and practices (KAPs) of the Cameroonian population on barrier and preventive measures against COVID-19; the first undertaken in the cities of Douala and Bangangté since the beginning of the COVID-19 pandemic. A cross-sectional study was carried out on 777 residents in the Littoral (Douala) and in the West (Banganté) regions of Cameroon from a structured, pretested and self-administered questionnaire (according to WHO guidelines). Data were collected and analyzed using SPSS version 18.0 software. For $\mathrm{p}=0.05$, the difference was statistically significant. Out of 777 participants, 67.7\% ( $\mathrm{n}=526$ ) were from Douala and 32.3\% (n=251) from Bangangté. Seventy percent $(70 \%)$ of our participants were aware of the main
\end{abstract}


preventive measures against COVID-19 taken by the Cameroonian government. The most used preventive method was the practice of hand hygiene $(88.9 \%)$; followed by social distancing $(87.2 \%)$, respiratory hygiene $(75.8 \%)$, and self-confinement $(85.3 \%)$. The majority of our participants had a positive attitude towards hand hygiene $(81.6 \%)$ and wearing a face mask (84.7\%). About $52.9 \%$ wore a face mask occasionally, $66.67 \%$ always washed their hands, $53.1 \%$ always used a hand sanitizer to disinfect their hands and $65,4 \%$ regularly self-confined themselves homes. Improving behaviors / attitudes and practices remain a major challenge for our populations. It is important to integrate the community in decision-making and increase awareness among our populations. Further studies are required to assess the impact of strategies undertaken by the decision makers, to roll out the COVID-19 pandemic in Cameroon.

Keywords: Knowledge, Attitudes, Practices, Barrier and Preventive Measures, COVID-19

\section{Introduction}

The year 2019 ended with the presence of a new strain of the corona virus which has not been previously identified in humans $[1,2]$ : The SARS-COV 2, which is responsible for a worldwide pandemic called "Coronavirus Disease 2019 (COVID-19)" [3, 4]. The first case was identified in Wuhan, located in the province of Hubei, China [5]. It became an international public health emergency on January 30, 2020, then a pandemic on March 11, 2020 with 137 countries reached on all the six continents so far [6].

It has been found that the SARS-COV 2 can be directly transmitted by droplets emitted by coughing or sneezing and /or by speaking $[7,8]$ and indirectly through soiled hands or surfaces in contact with the face $[8,9]$. The main signs and symptoms ( 70 to $80 \%$ of cases) of this disease are cough and fever according to several authors [8-11]. Minor symptoms such as fatigue, dyspnea, muscle pain, sore throat, nausea and vomiting may also be found [10]. The COVID-19 is most often an asymptomatic disease (in more than $85 \%$ ). However, while most people have mild symptoms, some people in the contrary might develop acute respiratory distress syndrome (ARDS) [10]. The COVID-19 doesn't yet have a therapeutic issue. Even if a vaccine is at hand [10], only simple and effective barriers methods developed by WHO may guarantee its prevention and control.

Studies assessing the knowledge attitudes and practices have been carried out across the world since the start of the pandemic [3-5, 12-14]. But, in Cameroon, there is a rarity of studies until now [15]. Furthermore, thirteen essential preventive measures have been put in place by the government throughout the national territory to curb the pandemic spread [16]. Assessing the knowledge, attitudes and practices of health professionals and populations is therefore a key tool of assessment of that prevention strategy. The aim of this study is to help in optimizing adherence to barrier and preventive measures of populations in order to break down the spread of COVID-19 in Cameroon.

\section{Methodology}

We conducted a cross-sectional study during eight months from March 20 to October 25, 2020. Data were collected through a structured, pre-tested and self-administered questionnaire among populations of Song Mahop (in Douala) and in Bangangté, both towns in Cameroon. The samples collection period lasted two months from May to July 2020.

In order to verify the well understanding of our questionnaire, we carried out a pretest with 30 participants in the New Bell District in Douala and 20 people in Bafoussam in the West region Cameroon. Our study population consisted of residents of the Song Mahop District, Douala and those of Bangangté. The minimum sample size was estimated at 422 participants from the Lorentz formula where we used a default prevalence of $50 \%$ with an estimated threshold of $5 \%$. We used a non-probabilistic and accidental sampling method to reach 777 respondents. Included in our study were all the inhabitants of the Song Mahop district in Douala and those of Bangangté aged more than 18 years and who had given their consent. Statistical analyses were performed using This Stat View 5.0 analysis software. The percentages of each variable were calculated as well as the Odds Ratio, the confidence intervals and the P-Value to find association between variables. We used Mari Josée Essie's evaluation grid to define the KAP evaluation score. Thus, the KAP were bad when the percentage was less than 60 , average between 60 and $80 \%$ and good at $80 \%$ and above. For p-value less than 0.05 , the difference was statistically significant.

Ethical Considerations

We obtained an institutional ethical clearance from the University of Douala (N ${ }^{\circ} 2394$ CEI-Udo/09/2020/M), an approval from authorities in Douala and Banganté. The signed informed consent of each participant was obtained and the confidentiality participant's data was respected.

\section{Results}

\subsection{Socio-demographic Characteristics of the Study Population}

Table 1 below depicts the distribution of populations by socio-demographic characteristics.

Out of 800 people surveyed, 777 (97.7\%) fully completed the questionnaire. Amongst them, 67.7\% (526/777) were in the urban area of Douala and $32.3 \%(251 / 777)$ in the rural area of Banganté. Male were slightly predominant $55.7 \%$ (433/777) and the majority were aged $18-29$ years (71.2\%). In addition, about half of our participants were unemployed 
(49.8\%) while $22.5 \%$ were engaged in informal activities. Majority of our participants $(49.3 \%)$ had a secondary level of

education compared to $4.9 \%$ who had never gone to school.

Table 1. Socio-demographic characteristics of respondents.

\begin{tabular}{|c|c|c|}
\hline Characteristics & Workforce (n) & Percentage (\%) \\
\hline \multicolumn{3}{|l|}{ Location } \\
\hline Douala & 526 & 67.7 \\
\hline Bangangte & 251 & 32.3 \\
\hline \multicolumn{3}{|l|}{ gender } \\
\hline Male & 433 & 55.7 \\
\hline Female & 344 & 44.3 \\
\hline \multicolumn{3}{|l|}{ Marital status } \\
\hline Single & 433 & 55.7 \\
\hline Married & 201 & 25.9 \\
\hline Free union & 110 & 14.1 \\
\hline Divorcee & 16 & 2.1 \\
\hline Widow (er) & 17 & 2.2 \\
\hline \multicolumn{3}{|l|}{ age range (Years) } \\
\hline ] $18 ; 29]$ & 553 & 71,2 \\
\hline ] 29; 39] & 126 & 16.2 \\
\hline ] 40; 49] & 62 & 8.0 \\
\hline ] 49; 59] & 24 & 3.1 \\
\hline$>60$ & 12 & 1.5 \\
\hline \multicolumn{3}{|c|}{ Type of employment } \\
\hline Informal & 175 & 22.5 \\
\hline Formal & 215 & 27.7 \\
\hline Unemployed & 387 & 49.8 \\
\hline \multicolumn{3}{|l|}{ Monthly income } \\
\hline ] $0 ; 36 \mathrm{k}]$ & 467 & 60.1 \\
\hline ]36k; 100k] & 179 & 23.0 \\
\hline ]100k; 200k] & 79 & 10.2 \\
\hline$>200 \mathrm{k}$ & 52 & 6.7 \\
\hline \multicolumn{3}{|l|}{ Study level } \\
\hline No & 38 & 4.9 \\
\hline Primary & 71 & 9.1 \\
\hline Secondary & 383 & 49,3 \\
\hline University & 285 & 36.7 \\
\hline \multicolumn{3}{|l|}{ Religion } \\
\hline Christian & 676 & 87.0 \\
\hline Muslim & 60 & 7.7 \\
\hline Animist & 34 & 4.4 \\
\hline Atheist & 7 & 0.9 \\
\hline
\end{tabular}

$1 \mathrm{k}=10^{3}$.

\subsection{Knowledge of Respondents About COVID-19}

Most of our respondents (70.7\%) were aware of the existence of COVID-19. The majority (83.8\%) knew the causal agent (viral origin) of the disease. Most of them $(76.3 \%)$ declared that the disease is transmitted by soiled hands and through droplets or aerosols from an infected patient who coughs, sneezes or speaks. Only 34.5\% (268/777) of respondents provided the response that the pandemic was not yet under control in Cameroon as of (date).

Respondents could correctly identify the symptoms of COVID-19 such as fever (83.8\%), cough (91.248\%), runny nose $(77.9 \%)$, sore throat $(69.6 \%)$, shortness of breath (66.7\%), and headache (72.2\%). Most of them (79.8\%) were aware that the COVID-19 patients most at risk of complications were older subjects. However, 79.4\% thought that everyone was at risk of contracting COVID-19.

Most of our respondents declared they practiced primary prevention methods against COVID-19, such as the practice of hand hygiene $(88.9 \%)$; social distancing $(87.3 \%)$, respiratory hygiene $(75.8 \%)$, and home confinement $(85.3 \%)$ converse to others who portrayed greater trust in vaccines (27.5\%), chloroquine (46.8\%), antibiotics (25.3\%), consumption of plants / concoctions $(53.3 \%)$ and hot water $(50.8 \%)$ as valid tools in the prevention of COVID-19. More than $70 \%$ of our respondents were aware of the thirteen barriers measures put in place by Cameroonian government to fight again spread the COVID-19 (table 2). 
Table 2. Distribution of respondents according to their knowledge on COVID-19.

\begin{tabular}{|c|c|c|}
\hline Knowledge of respondents & Correct answer $\mathbf{n}(\%)$ & Wrong answer $\mathrm{n}(\%)$ \\
\hline \multicolumn{3}{|l|}{ What do you know about COVID-19? } \\
\hline COVID-19 is a disease made in the laboratory & $309(39.8 \%)$ & $468(60.2 \%)$ \\
\hline COVID-19 does not exist & $549(70.7 \%)$ & $228(29.3 \%)$ \\
\hline COVID-19 is a mystical disease & $592(76.2 \%)$ & $185(23.8 \%)$ \\
\hline COVID-19 is a disease caused by a virus & $616(79.3 \%)$ & $161(20.7 \%)$ \\
\hline COVID-19 is transmitted by mosquitoes & $651(83.8 \%)$ & $126(16.2 \%)$ \\
\hline COVID-19 is transmitted through soiled hands & $593(76.3 \%)$ & $184(23.7 \%)$ \\
\hline COVID-19 is spread by droplets or aerosols from a patient who coughs, sneezes or speaks & $701(90.2 \%)$ & $76(9.8 \%)$ \\
\hline There is already a treatment for COVID 19 & $85(10.9 \%)$ & $692(89.1 \%)$ \\
\hline It is a pandemic already under control in Cameroon & $268(34.5 \%)$ & $509(65.5 \%)$ \\
\hline \multicolumn{3}{|l|}{ Symptoms of COVID-19 } \\
\hline Fever & $651(83.8 \%)$ & $126(16.2 \%)$ \\
\hline Cough & $709(91.2 \%)$ & $68(8.7 \%)$ \\
\hline Running nose & $605(77.9 \%)$ & $172(22.1 \%)$ \\
\hline Sore throat & $541(69.6 \%)$ & $236(30.4 \%)$ \\
\hline Diarrhea & $592(76.2 \%)$ & $185(23.8 \%)$ \\
\hline Shortness of breath & $518(66.7 \%)$ & $259(33.3 \%)$ \\
\hline Aches & $268(34.5 \%)$ & $509(65.5 \%)$ \\
\hline Headache & $561(72.2 \%)$ & $216(27.8 \%)$ \\
\hline \multicolumn{3}{|l|}{ The most exposed are: } \\
\hline Senior citizens & $620(79.8 \%)$ & $157(20.2 \%)$ \\
\hline Young people & $515(66.3 \%)$ & $262(33.7 \%)$ \\
\hline Men & $463(59.6 \%)$ & $314(40.4 \%)$ \\
\hline The women & $461(59.3 \%)$ & $316(40.7 \%)$ \\
\hline The rich & $453(58.3 \%)$ & $324(41.7 \%)$ \\
\hline Poor people & $465(59.8 \%)$ & $312(40.1 \%)$ \\
\hline Everybody & $160(20.6 \%)$ & $617(79.4 \%)$ \\
\hline \multicolumn{3}{|l|}{ The means of prevention of COVID-19 are based on: } \\
\hline The vaccine & $563(72.4 \%)$ & $214(27.5 \%)$ \\
\hline Chloroquine & $413(53.1 \%)$ & $364(46.8 \%)$ \\
\hline Antibiotics & $580(74.6 \%)$ & $197(25.3 \%)$ \\
\hline Plants / bark / concoctions & $363(46.7 \%)$ & $414(53.3 \%)$ \\
\hline Hot water & $382(49.2 \%$ & $395(50.8 \%)$ \\
\hline Hand hygiene & $691(88.9 \%)$ & $86(11.1 \%)$ \\
\hline Social distancing & $678(87.2 \%)$ & $99(12.7 \%)$ \\
\hline Respiratory hygiene & $589(75.8 \%)$ & $188(24.2 \%)$ \\
\hline Stay at home & $663(85.3 \%)$ & $114(14.7 \%)$ \\
\hline \multicolumn{3}{|c|}{ Are the following measures part of the 13 measures recommended in Cameroon against COVID-19? } \\
\hline The closure of all schools from kindergarten to higher education & $744(95.7 \%)$ & $33(4.2 \%)$ \\
\hline The closure of drinking establishments, restaurants and leisure areas & $710(91.4 \%)$ & $67(8.6 \%)$ \\
\hline Regulation of consumer flows in markets and shopping centers & $621(79.9 \%)$ & $156(20.1 \%)$ \\
\hline Prohibition of overloading in public transport agencies & $700(90.2 \%)$ & $77(9.8 \%)$ \\
\hline Use of electronic means of communication and digital tools for meetings of at least 10 people & $608(78.2 \%)$ & $169(21.7 \%)$ \\
\hline Avoiding social contact (shaking hands, hugging) & $722(92.9 \%)$ & $55(7.1 \%)$ \\
\hline Strict adherence to hand hygiene measures & $722(92.9 \%)$ & $55(7.1 \%)$ \\
\hline Closure of national borders & $709(91.2 \%)$ & $68(8.7 \%)$ \\
\hline Reporting of school and university competitions later & $716(92.1 \%)$ & $61(7.8 \%)$ \\
\hline Ban on gatherings of more than 50 people & $715(92.0 \%)$ & $62(8.0 \%)$ \\
\hline
\end{tabular}

\subsection{Respondents' Attitudes Towards COVID-19}

In this study, we assessed the attitudes of our respondents. We noted that $81.6 \%$ of our respondents agreed that everyone should comply with hand hygiene recommendations versus $10.8 \%$ who were neutral. The majority $(84.7 \%)$ agreed that everyone should wear a face mask. Surprisingly, $69.1 \%$
(537/777) respondents did not agree to benefit from an experimental vaccine against COVID-19.

Most of respondents (66.5\%) argued that an infection with COVID-19 can be cured compared to $15.4 \%$ who disagreed. In case of the COVID-19 symptoms, $65.2 \%$ of the participants agreed to call the SOS-free number and $15.8 \%$ were reluctant to do so. Also, $80.2 \%$ of respondents agreed to 
convince all suspicious people to call the free call center (SOS number) versus 9.6\% who did not agree.

Table 3. The attitudes of populations towards COVID-19.

\begin{tabular}{|c|c|c|c|}
\hline ATTITUDES & Okay & Neutral & Disagree \\
\hline \multicolumn{4}{|l|}{ Who Should Follow Hand Hygiene Recommendations? } \\
\hline Only nursing staff & $151(19.4 \%)$ & $84(10.8 \%)$ & $542(69.7 \%)$ \\
\hline All the sick & $358(46.1 \%)$ & $78(10.0 \%)$ & $341(43.9 \%)$ \\
\hline COVID-19 patients only & $163(21.0 \%)$ & $80(10.3 \%)$ & $534(68.7 \%)$ \\
\hline Everybody & $634(81.6 \%)$ & $30(3.9 \%)$ & $113(14.5 \%$ \\
\hline Nobody & $127(16.3 \%)$ & $102(13.1 \%)$ & $548(70.5 \%)$ \\
\hline \multicolumn{4}{|l|}{ Who should wear a face mask? } \\
\hline Only nursing staff & $118(15.2 \%)$ & $96(12.3 \%)$ & $56372.4 \%)$ \\
\hline All the sick & $407(52.4 \%)$ & $72(9.3 \%)$ & $298(38.3 \%)$ \\
\hline Corona / COVID-19 patients only & $178(23 \%)$ & $86(11.1 \%)$ & $513(66.0 \%)$ \\
\hline Everybody & $658(84.7 \%)$ & $42(5.4 \%)$ & $77(9.9 \%)$ \\
\hline Nobody & $122(15.7 \%)$ & $94(12.1 \%)$ & $561(72.2 \%)$ \\
\hline \multicolumn{4}{|l|}{ About the vaccine } \\
\hline Would you agree to benefit from an experimental vaccine against COVID-19? & $125(16.1 \%)$ & $115(14.8 \%)$ & $537(69.1 \%)$ \\
\hline I will not agree to be vaccinated because it is to contaminate or sterilize the population & $264(49.2 \%)$ & $138(25.7 \%)$ & $135(25.1 \%)$ \\
\hline I will not agree to be vaccinated because it is pharmaceutical lobbying & $196(36.5 \%)$ & $160(29.8 \%)$ & $181(33.7 \%)$ \\
\hline I will not agree to be vaccinated because I do not want to be the guinea pig of the pharmaceutical companies & $338(62.9 \%)$ & $74(13.8 \%)$ & $125(23.3 \%)$ \\
\hline I will not agree to be vaccinated because it is useless & $194(36.1 \%)$ & $152(28.3 \%)$ & $191(35.6 \%)$ \\
\hline \multicolumn{4}{|l|}{ Patients with COVID-19 are } \\
\hline Sick for life & $208(26.8 \%)$ & $114(14.7 \%)$ & $455(58.5 \%)$ \\
\hline Patients who will keep the consequences & $210(27.0 \%)$ & $181(23.3 \%)$ & $386(49.7 \%)$ \\
\hline Patients who will be able to heal completely & $517(66.5 \%)$ & $140(18.0 \%)$ & $120(15.4 \%)$ \\
\hline Patients who will surely die & $205(26.4 \%)$ & $177(22.8 \%)$ & $395(50.8 \%)$ \\
\hline \multicolumn{4}{|l|}{ Attitude to the evocative signs of COVID-19? } \\
\hline Call the toll-free number if I feel the suggestive symptoms & $507(65.2 \%)$ & $123(15.8 \%)$ & $147(18.9 \%)$ \\
\hline Convince all suspicious people to call the toll free number & $623(80.2 \%)$ & $79(10.2 \%)$ & $75(9.6 \%)$ \\
\hline Wait for the disease to get worse, then I call the toll free number & $117(15.0 \%)$ & $77(9.9 \%)$ & $583(75.0 \%)$ \\
\hline Stay away from anyone suspicious & $514(66.1 \%)$ & $79(10.2 \%)$ & $187(23.7 \%)$ \\
\hline Refer any suspicious person to a traditional practitioner & $107(13.8 \%)$ & $185(23.8 \%)$ & $485(62.4 \%)$ \\
\hline The suspected case does not concern me, so I do nothing & $77(9.9 \%)$ & $94(12.1 \%)$ & $606(78 \%)$ \\
\hline
\end{tabular}

\subsection{Respondents' Practices Towards COVID-19}

In this study, we investigated the practice of barrier and preventive measures against COVID-19. We found that $52.9 \%$ of respondents said they wear a face mask occasionally against $2.4 \%$ who declared to have never wore it. The majority $(66.7 \%)$ always washed their hands compared to $1.5 \%$ who never practiced it. About half of our respondents declared using a hydro-alcoholic solution to disinfect their hands and always avoiding touching their nose, mouth, eyes with soiled hands $(53.1 \%$ and $56.0 \%$ respectively).

More than half of respondents $(63.1 \%)$ revealed always sneezing, coughing in the fold of the elbow or in a disposable tissue and also, avoiding contact greetings (68.1\%). Most of our surveyed population $(61.6 \%)$ always refrained from visiting crowded places and kept children at home since the closure of schools and universities (73.4\%).

Table 4. Distribution of respondents according to their practices.

\begin{tabular}{|c|c|c|c|}
\hline PRACTICE & Always & Occasionally & Never \\
\hline Are you wearing the mask (face mask)? & $347(44.7 \%)$ & $411(52.9 \%)$ & $19(2.4 \%)$ \\
\hline Do you wash your hands with running water and soap? & $518(66.7 \%)$ & $247(31.8 \%)$ & $12(1.5 \%)$ \\
\hline Do you have a hydro-alcoholic solution that you use to disinfect your hands? & $413(53.1 \%)$ & $318(40.9 \%)$ & $46(5.9 \%)$ \\
\hline Do you avoid touching your nose, mouth and eyes when your hands are soiled? & $435(56.0 \%)$ & $305(39.2 \%)$ & $37(4.8 \%)$ \\
\hline Do you sneeze and cough into the crease of your elbow or into a disposable single use tissue? & $490(63.1 \%)$ & $248(31.9 \%)$ & $39(5.0 \%)$ \\
\hline Do you avoid contact greetings (shaking hands, hugs)? & $529(68.1 \%)$ & $209(26.9 \%)$ & $39(5.0 \%)$ \\
\hline $\begin{array}{l}\text { I refrain from visiting places crowded with people (meetings, bereavements, weddings, restaurant, bar, } \\
\text { church / mosque) }\end{array}$ & $479(61.6 \%)$ & $248(31.9 \%)$ & $50(6.4 \%)$ \\
\hline I stay at home and only go out when necessary & $508(65.4 \%)$ & $201(25.9 \%)$ & $68(8.7 \%)$ \\
\hline All children have been held at home since schools and universities closed & $570(73.3 \%)$ & $167(21.5 \%)$ & $40(5.1 \%)$ \\
\hline The entourage influences my good practices & $387(49.8 \%)$ & $185(23.8 \%)$ & $205(26.4 \%)$ \\
\hline
\end{tabular}




\subsection{Identification of the Determinants of the Attitude Towards a Potential Vaccine Against COVID-19}

In this study, we looked for the determinants of the attitude of populations towards a potential vaccine through a multivariate analysis. Compared to respondents with no level of education, more respondents with a primary level of education had declared to agree an experimental vaccine against COVID-19 (25.3\% vs. 10.5\%). This association was statistically significant in multivariate polytonic logistic regression $(\mathrm{ORa}=5.77,[1.26-26.43], \mathrm{P}=0.02)$.
The average knowledge score for respondents who said they disagreed to benefit from a potential COVID-19 vaccine was $72.1 \pm 10.1$ compared to $69.7 \pm 12.2$ among those with a neutral opinion. In multivariate polytonic logistic regression, this difference was statistically significant $(\mathrm{ORa}=1.02,[1.00$ $1.04], \mathrm{P}=0.02$ ). In addition, the average knowledge score by respondents who agreed to benefit from a potential COVID19 vaccine was $68.63 \pm 1.68$ versus $69.71 \pm 12.2$ among those with a neutral opinion, $\mathrm{p}=0.67$.

Table 5. Determinants of the attitude towards a potential vaccine against COVID-19 in polytonic logistic regression according to the multivariate model.

\begin{tabular}{|c|c|c|c|c|c|}
\hline & \multicolumn{5}{|c|}{ Would you agree to benefit from an experimental vaccine against COVID-19? } \\
\hline & Total $(\mathrm{N}=777)$ & Disagree & ORa, 95\% CI, p-value & Okay & ORa, 95\% CI, p-value \\
\hline \multicolumn{6}{|l|}{ Location } \\
\hline Douala & 526 & $363(69.0 \%)$ & - & $82(15.6 \%)$ & - \\
\hline Bangangte & 251 & $174(69.3 \%)$ & $\mathrm{ORa}=1.19,[0.73-1.92], \mathrm{P}=0.49$ & $43(17.1 \%)$ & $\mathrm{ORa}=1.27,[0.70-2.30], \mathrm{P}=0.44$ \\
\hline \multicolumn{6}{|l|}{ Gender } \\
\hline Male & 432 & $295(68.3 \%)$ & - & $72(16.7 \%)$ & - \\
\hline Female & 344 & $242(70.3 \%)$ & $\mathrm{ORa}=1.03,[0.67-1.58], \mathrm{P}=0.89$ & $53(15.4 \%)$ & $\mathrm{ORa}=0.97,[0.57-1.67], \mathrm{P}=0.92$ \\
\hline Single & 433 & $296(68.4 \%)$ & - & $72(16.6 \%)$ & - \\
\hline Married & 201 & $144(71.6 \%)$ & $\mathrm{ORa}=1.43,[0.78-2.64], \mathrm{P}=0.25$ & $30(14.9 \%)$ & $\mathrm{ORa}=0.99,[0.46-2.15], \mathrm{P}=1.00$ \\
\hline Free Union & 110 & $75(68.2 \%)$ & $\mathrm{ORa}=1.04,[0.56-1.93], \mathrm{P}=0.91$ & $18(16.4 \%)$ & $\mathrm{ORa}=0.94,[0.43-2.05], \mathrm{P}=0.88$ \\
\hline Divorce (e) or widow (er) & 33 & $22(66.7 \%)$ & $\mathrm{ORa}=1.13,[0.38-3.38], \mathrm{P}=0.82$ & $5(15.1 \%)$ & $\mathrm{ORa}=0.78,[0.19-3.25], \mathrm{P}=0.73$ \\
\hline \multicolumn{6}{|l|}{ Monthly income } \\
\hline$[0 ; 36000]$ & 467 & $328(70.3 \%)$ & - & $76(16.3 \%)$ & - \\
\hline$[36000 ; 100,000]$ & 179 & $115(64.2 \%)$ & $\mathrm{ORa}=0.68,[0.40-1.16], \mathrm{P}=0.16$ & $32(17.9 \%)$ & $\mathrm{ORa}=0.72,[0.37-1.40], \mathrm{P}=0.33$ \\
\hline$>200,000$ & 52 & $33(63.5 \%)$ & $\mathrm{ORa}=0.66,[0.28-1.51], \mathrm{P}=0.32$ & $9(17.3 \%)$ & $\mathrm{ORa}=0.75,[0.26-2.17], \mathrm{P}=0.60$ \\
\hline \multicolumn{6}{|l|}{ School level } \\
\hline No & 38 & $26(68.4 \%)$ & - & $4(10.5 \%)$ & - \\
\hline Primary & 71 & $46(64.8 \%)$ & $\mathrm{ORa}=2.47,[0.77-7.88], \mathrm{P}=0.13$ & $18(25.3 \%)$ & $\mathrm{ORa}=5.77,[1.26-26.43], \mathrm{P}=0.02$ \\
\hline Secondary & 383 & $268(70.0 \%)$ & $\mathrm{ORa}=1.64,[0.67-3.98], \mathrm{P}=0.28$ & $60(15.7 \%)$ & $\mathrm{ORa}=2.67,[0.73-9.77], \mathrm{P}=0.14$ \\
\hline University & 285 & $197(69.1 \%)$ & $\mathrm{ORa}=1.39,[0.56-3.45], \mathrm{P}=0.48$ & $43(15.1 \%)$ & $\mathrm{ORa}=2.38,[0.6-8.97], \mathrm{P}=0.20$ \\
\hline \multicolumn{6}{|l|}{ Religion } \\
\hline Christian & 676 & $472(69.8 \%)$ & - & $105(15.5 \%)$ & - \\
\hline Muslim & 60 & $37(61.7 \%)$ & $\mathrm{ORa}=0.87,[0.40-1.91], \mathrm{P}=0.73$ & $14(23.3 \%)$ & $\mathrm{ORa}=1.50,[0.61-3.73], \mathrm{P}=0.38$ \\
\hline Animist & 34 & $23(67.6 \%)$ & $\mathrm{ORa}=0.84,[0.32-2.17], \mathrm{P}=0.71$ & $5(14.7 \%)$ & $\mathrm{ORa}=0.70,[0.20-2.45, \mathrm{P}=0.58$ \\
\hline Atheist & 7 & $5(71.4 \%)$ & $\mathrm{ORa}=1.06,[0.11-9.95], \mathrm{P}=0.96$ & $1(14.3 \%)$ & $\mathrm{ORa}=1.13,[0.06-20.31], \mathrm{P}=0.93$ \\
\hline \multicolumn{6}{|c|}{ Knowledge score ( $\%$ correct answers) } \\
\hline Average (DS) & & $72.1(10.1)$ & $\mathrm{ORa}=1.02,[1.004-1.04], \mathrm{P}=0.02$ & $68.6(10.7)$ & $\mathrm{ORa}=0.99,[0.97-1.02], \mathrm{P}=0.66$ \\
\hline \multicolumn{6}{|l|}{ Age (Years) } \\
\hline Average (DS) & & $31.5(10.6)$ & $\mathrm{ORa}=0.99[0.97-1.02], \mathrm{P}=0.53$ & $32.1(12.3)$ & $\mathrm{ORa}=1.01,[0.98-1.04], \mathrm{P}=0.56$ \\
\hline
\end{tabular}

Table 6. Determinants of wearing a face mask in polytonic logistic regression *: multivariate model.

\begin{tabular}{|c|c|c|c|c|c|}
\hline \multirow{2}{*}{ PRACTICE } & \multicolumn{5}{|c|}{ Are you wearing a face mask? } \\
\hline & Total $(\mathbf{N}=777)$ & Never & ORa, $95 \%$ CI, p-value & Always & ORa, 95\% CI, p-value \\
\hline \multicolumn{6}{|l|}{ Location } \\
\hline Douala & 526 & $13(2.5 \%)$ & - & $277(52.7 \%)$ & - \\
\hline Bangangte & 251 & $6(2.4 \%)$ & ORa: $0.675,[0.223-2.045], \mathrm{P}=0.48$ & $69(27.5 \%)$ & ORa: $0.28,[0.19-0.41], \mathrm{P}<0.0001$ \\
\hline \multicolumn{6}{|l|}{ Sex } \\
\hline Male & 432 & $9(20.1 \%)$ & - & $179(41.4 \%)$ & - \\
\hline Female & 344 & $10(2.9 \%)$ & ORa: $1.872,[0.691-5.067], \mathrm{P}=0.21$ & $167(48.5 \%)$ & ORa: $1.37,[0.1002-1.88], \mathrm{P}=0.04$ \\
\hline \multicolumn{6}{|l|}{ Age (year) } \\
\hline Average (DS) & $31.71(11)$ & $31.74(13.2)$ & ORa: $0.993,[0.936-1.053], \mathrm{P}=0.81$ & $31.33(10.6)$ & ORa: $1.01,[0.99-1.03], \mathrm{P}=0.27$ \\
\hline \multicolumn{6}{|l|}{ Marital status } \\
\hline Single & 433 & $9(2 \%)$ & - & $202(46.6 \%)$ & - \\
\hline Married & 201 & $6(3.0 \%)$ & ORa: $1.21,[0.32-4.54], \mathrm{P}=0.76$ & $81(40.3 \%)$ & ORa: $0.73,[0.47-1.16], \mathrm{P}=0.18$ \\
\hline Free Union & 110 & $3(2.7 \%)$ & ORa: $1.002,[0.24-4.158], \mathrm{P}=0.99$ & $45(40.9 \%)$ & ORa: $0.68,[0.42-1.09], \mathrm{P}=0.11$ \\
\hline Divorce (e) or widow (er) & 33 & $1(3.0 \%)$ & ORa: $1.028,[0.080-13.269], \mathrm{P}=0.98$ & $18(54.5 \%)$ & ORa: $097,[0.41-2.30], \mathrm{P}=0.95$ \\
\hline \multicolumn{6}{|l|}{ Type of employment } \\
\hline Informal & 175 & $5(2.9 \%)$ & - & $69(39.4 \%)$ & \\
\hline
\end{tabular}




\begin{tabular}{|c|c|c|c|c|c|}
\hline \multirow{2}{*}{ PRACTICE } & \multicolumn{5}{|c|}{ Are you wearing a face mask? } \\
\hline & Total $(\mathrm{N}=777)$ & Never & ORa, $95 \%$ CI, p-value & Always & ORa, 95\% CI, p-value \\
\hline Formal & 215 & $6(2.8 \%)$ & ORa: $1.55,[0.39-6.125], \mathrm{P}=0.53$ & $93(43.3 \%)$ & ORa: $1.21,[0.75-1.97], \mathrm{P}=0.44$ \\
\hline Unemployed & 387 & $8(2.1 \%)$ & ORa: $1.004,[0.23-4.243], \mathrm{P}=0.99$ & $184(47.5 \%)$ & ORa: $1.17,[0.73-1.89], \mathrm{P}=0.45$ \\
\hline \multicolumn{6}{|l|}{ Monthly income } \\
\hline ] $0 ; 36000]$ & 467 & $10(2.1 \%)$ & - & $213(45.6 \%)$ & - \\
\hline ] $36000 ; 100,000]$ & 179 & $6(3.3 \%)$ & ORa: $1.51,[0.39-5.89], \mathrm{P}=0.54$ & $71(39.7 \%)$ & ORa: $1.32,[0.97-1.81], \mathrm{P}=0.91$ \\
\hline ] 100,$000 ; 200000]$ & 79 & $2(2.5 \%)$ & ORa: $1.16,[0.17-7.725], \mathrm{P}=0.87$ & $35(44.3 \%)$ & ORa: $1.32,[0.97-1.81], \mathrm{P}=0.07$ \\
\hline$>200,000$ & 52 & $1(1.9 \%)$ & ORa: $1.16,[0.109-12.34], \mathrm{P}=0.90$ & $27(51.9 \%)$ & ORa: $1.32,[0.97-1.81], \mathrm{P}=0.07$ \\
\hline \multicolumn{6}{|l|}{ Study level } \\
\hline No & 38 & $2(5.3 \%)$ & - & $19(\%)$ & - \\
\hline Primary & 71 & $1(1.41 \%)$ & ORa: 0.146, [0.011-1.900], $\mathrm{P}=0.14$ & $28(\%)$ & ORa: $0.72,[0.30-1.73], \mathrm{P}=0.46$ \\
\hline Secondary & 383 & $11(2.9 \%)$ & ORa: $0.42,[0.77-2.29], \mathrm{P}=0.31$ & $168(\%)$ & ORa: $0.56,[0.26-1.18], \mathrm{P}=0.13$ \\
\hline University & 285 & $5(1.7 \%)$ & ORa: $0.24,[0.38-1.49], \mathrm{P}=0.12$ & $131(\%)$ & ORa: $0.73,[0.34-1.57], \mathrm{P}=0.42$ \\
\hline \multicolumn{6}{|l|}{ Religion } \\
\hline Christian & 676 & $16(2.4 \%)$ & - & $298(44.1 \%)$ & - \\
\hline Muslim & 60 & $1(1.7 \%)$ & ORa: 0.67 [0.08-5.439], $\mathrm{P}=0.708$ & $30(50 \%)$ & ORa: $1.24,[0.70-2.183], \mathrm{P}=0.45$ \\
\hline Animist & 34 & $1(3.0 \%)$ & ORa: $1.71,[0.201-14.644], \mathrm{P}=0.62$ & $16(47.1 \%)$ & ORa: $1.37,[0.65-2.94], \mathrm{P}=0.41$ \\
\hline Athe & 7 & $1(14.2 \%)$ & ORa: $7.24,[0.54-96.043], \mathrm{P}=0.13$ & $3(42.9 \%)$ & ORa: $1.13,[0.21-6.04], \mathrm{P}=0.89$ \\
\hline \multicolumn{6}{|c|}{ COVID-19 Knowledge Score } \\
\hline Average (DS) & $71.22(10.6)$ & $67.93(12.1)$ & ORa: $0.97,[0.93-1.016], \mathrm{P}=0.219$ & $71.7(10.6)$ & ORa: $1.01,[0.99-1.02], \mathrm{P}=0.22$ \\
\hline
\end{tabular}

Table 7. Identification of the determinants of confinement in polytonic logistic regression *: multivariate model.

\begin{tabular}{|c|c|c|c|c|c|}
\hline \multirow{2}{*}{ PRACTICE } & \multicolumn{5}{|c|}{ I stay at home and only go out in case of necessity? } \\
\hline & Total $(\mathrm{N}=777)$ & Never & OR, 95\% CI, p-value & Always & OR, 95\% CI, p-value \\
\hline \multicolumn{6}{|l|}{ Location } \\
\hline Douala & 526 & $39(7.4 \%)$ & - & $349(66.3 \%)$ & - \\
\hline Bangangte & 251 & $29(11.5 \%)$ & $\mathrm{ORa}=1.47,[0.78-2.79], \mathrm{P}=0.23$ & $159(63.3 \%)$ & $\mathrm{ORa}=0.90,[0.60-1.34], \mathrm{P}=0.60$ \\
\hline \multicolumn{6}{|c|}{ 然 } \\
\hline Male & 432 & $44(10.2 \%)$ & - & $262(60.6 \%)$ & - \\
\hline Female & 344 & $24(7.0 \%)$ & $\mathrm{ORa}=1.04,[0.56-1.92], \mathrm{P}=0.90$ & $245(71.2 \%)$ & $\mathrm{ORa}=1.89,[1.31-2.72], \mathrm{P}=0.007$ \\
\hline \multicolumn{6}{|l|}{ Age (year) } \\
\hline Average (DS) & $31.71(11)$ & $33.40(9.1)$ & $\mathrm{ORa}=1.02,[0.98-1.05], \mathrm{P}=0.30$ & $31.51(11.4)$ & $\mathrm{ORa}=1.02,[1.00-1.05], \mathrm{P}=0.05$ \\
\hline \multicolumn{6}{|l|}{ Marital status } \\
\hline Single & 433 & $35(8.1 \%)$ & - & $303(70.0 \%)$ & - \\
\hline Married & 201 & $20(9.9 \%)$ & $\mathrm{ORa}=0.61,[0.26-1.41], \mathrm{P}=0.25$ & $123(61.2 \%)$ & $\mathrm{ORa}=0.49,[0.29-0.83], \mathrm{P}=0.008$ \\
\hline Free Union & 110 & $10(9.1 \%)$ & $\mathrm{ORa}=0.75,[0.32-1.80], \mathrm{P}=0.52$ & $62(56.4 \%)$ & $\mathrm{ORa}=0.50,[0.30-0.83], \mathrm{P}=0.008$ \\
\hline Divorce (e) or widow (er) & 33 & $13(9.1 \%)$ & $\mathrm{ORa}=0.59,[0.12-2.91], \mathrm{P}=0.43$ & $82(57.3 \%)$ & $\mathrm{ORa}=0.37,[1.40-0.98], \mathrm{P}=0.04$ \\
\hline \multicolumn{6}{|l|}{ Type of employment } \\
\hline Informal & 175 & $20(11.4 \%)$ & - & $88(50.3 \%)$ & - \\
\hline Formal & 215 & $19(8.8 \%)$ & $\mathrm{ORa}=1.07,[0.47-2.48], \mathrm{P}=0.86$ & $149(69.3 \%)$ & $\mathrm{ORa}=1.99,[1.18-3.36], \mathrm{P}=0.009$ \\
\hline Unemployed & 387 & $29(7.5 \%)$ & $\mathrm{ORa}=1.30,[0.58-2.94], \mathrm{P}=0.53$ & $271(70.0 \%)$ & $\mathrm{ORa}=2.45,[1.47-4.09], \mathrm{P}=0.0006$ \\
\hline \multicolumn{6}{|l|}{ Monthly income } \\
\hline$[0 ; 36000]$ & 467 & $39(8.3 \%)$ & - & $310(66.4 \%)$ & - \\
\hline$[36000 ; 100,000]$ & 179 & $17(9.5 \%)$ & $\mathrm{ORa}=1.041,[0.45-2.38], \mathrm{P}=0.92$ & $106(59.2 \%)$ & $\mathrm{ORa}=1.21,[0.72-2.05], \mathrm{P}=0.47$ \\
\hline$[100,000 ; 200000]$ & 79 & $9(11.4 \%)$ & $\mathrm{ORa}=1.49,[0.48-4.59], \mathrm{P}=0.48$ & $54(68.3 \%)$ & $\mathrm{ORa}=1.43,[0.67-3.04], \mathrm{P}=0.35$ \\
\hline$>200,000$ & 52 & $3(5.8 \%)$ & $\mathrm{ORa}=0.63,[0.14-2.84], \mathrm{P}=0.55$ & $38(73.1 \%)$ & $\mathrm{ORa}=1.44,[0.61-3.41], \mathrm{P}=0.40$ \\
\hline \multicolumn{6}{|l|}{ Study level } \\
\hline No & 38 & $10(26.3 \%)$ & - & $22(57.9 \%)$ & - \\
\hline Primary & 71 & $5(7.0 \%)$ & $\mathrm{ORa}=0.13,[0.30-0.56], \mathrm{P}=0.006$ & $45(63.4 \%)$ & $\mathrm{ORa}=0.65,[0.22-1.93], \mathrm{P}=0.43$ \\
\hline Secondary & 383 & $27(7.0 \%)$ & $\mathrm{ORa}=0.15,[0.05-0.48], \mathrm{P}=0.014$ & $244(63.7 \%)$ & $\mathrm{ORa}=0.52,[0.19-1.37], \mathrm{P}=0.18$ \\
\hline University & 285 & $26(9.1 \%)$ & $\mathrm{ORa}=0.24,[0.07-0.79], \mathrm{P}=0.02$ & $197(69.1 \%)$ & $\mathrm{ORa}=0.68,[0.25-1.85], \mathrm{P}=0.45$ \\
\hline \multicolumn{6}{|l|}{ Religion } \\
\hline Christian & 676 & $59(8.7 \%)$ & - & $438(64.8 \%)$ & - \\
\hline Muslim & 60 & $6(10.0 \%)$ & $\mathrm{ORa}=1.44,[0.50-4.15], \mathrm{P}=0.50$ & $41(68.3 \%)$ & $\mathrm{ORa}=1.62,[0.82-3.20], \mathrm{P}=0.16$ \\
\hline Animist & 34 & $2(5.9 \%)$ & $\mathrm{ORa}=1.14,[0.22-5.79], \mathrm{P}=0.88$ & $25(73.5 \%)$ & $\mathrm{ORa}=2.31,[0.94-5.70], \mathrm{P}=0.07$ \\
\hline Atheist & 7 & $1(14.3 \%)$ & $\mathrm{ORa}=1.80,[0.14-23.93], \mathrm{P}=0.65$ & $4(57.1 \%)$ & $\mathrm{ORa}=1.37,[0.21-8.74], \mathrm{P}=0.74$ \\
\hline \multicolumn{6}{|c|}{ COVID-19 Knowledge Score } \\
\hline Average (DS) & $71.22(10.61)$ & $68.88(9.7)$ & $\mathrm{ORa}=0.99,[0.96-1.01], \mathrm{P}=0.40$ & $72.1(10.3)$ & $\mathrm{ORa}=1.02,[1.004-1.04], \mathrm{P}=0.01$ \\
\hline * Dependent variable ref & rence: " Occasion & & & & \\
\hline
\end{tabular}

\subsection{Identification of the Determinants of Wearing a Face Mask}

In this study, there was a better usage of face masks in
Douala when compared to Bangangte $(52.7 \%$ vs. $27.5 \%$, ORa: 0.28, [0.19-0.41], P <0.0001). Similarly, more men, wore facemasks compared to females $(48.5 \%$ vs. $41.4 \%$, ORa: $1.37,[0.1002-1.88], \mathrm{P}=0.04)$ (table 6). 


\subsection{The Determinants of Confinement}

We noted that more women than men self-confined $(71.2 \%$ versus $60.6 \%, \mathrm{ORa}=1.89, \quad[1.31-2.72], \quad \mathrm{P}=0.007)$. Also, married $(61.2 \%)$, people of the formal sector $(70.0 \%)$ were the most confined at home $(\mathrm{ORa}=1.99,[1.18-3.36], \mathrm{P}=0.009$; $\mathrm{ORa}=2.45,[1.47-4.09], \mathrm{P}=0.0006)$ than other groups of people.

\section{Discussion}

Out of 800 people targeted, $777(97.7 \%)$ filled the questionnaire following the prescribed guides. amongst which $67.7 \%(526 / 777)$ lived in Douala urban area while $32.3 \%$ $(251 / 777)$ in Banganté rural area. The age of respondents ranged from 18-29 years; the male gender was predominance in both the urban and rural setting $(71.2 \%$ and $55.7 \%$ respectively). Several studies across the world found similar results of male predominance amongst respondents [16-19] for instance in China (54\%) [17], 59.6\% in Nigeria [18], 57.9\% in India [19], 56\% in the South-West region of Cameroon [16]. However, other studies rather found a majority in female respondents [20] and 58.9\% in Pakistan [21]; 92.7\% at the Philippines [22], 76.6\% in Serbia [23]. This predominance in male participation to this study could be explained by implementation of home confinement which keeps men in their homes. We also think the sudden onset of the pandemic that resulted in record deaths across the globe made our study a "hot topic" and increased both participation to the study and overall awareness about the disease.

In our study, the most represented age group was 18 to 29 (71.2\%). Similar findings have been reported in Serbia [23], Egypt, [15], and Indonesia [24]. This age bracket superposes that the demography of Cameroon. Furthermore, in terms of level of education, the majority of respondents had secondary education (49.3\%). Paradoxically, the majority of respondents in a similar study from the South-West (Buea) region of Cameroon were university students and graduates $(55.6 \%)$ [16]. In fact, Buea is known as a university town.

Most of our respondents knew that COVID-19 exists (70.7\%). In a similar study in Pakistan, $80 \%$ of respondents were aware of the existence of COVID-19 [22]. Most of our respondent could identify the origin of the disease $(83.8 \%)$, its modes of transmission such as by soiled hands (76.3\%), by nasal droplets or saliva $(90.2 \%)$ and, even the associated signs and symptoms of the disease $(70 \%)$. These results are similar to those of LIU et al who had respondents identifying a droplet transmission at $98.9 \%$ and soiled hands at $0.3 \%$ [25]. Similarly, Narayana et al described $90.8 \%$ of the transmission through droplets in India among elderly and respondents with chronic diseases such as high blood pressure, cardiovascular and respiratory diseases $[11,19,26]$. Furthermore, in this study, we found that $79.4 \%$ believed that everyone was at risk of contracting severe forms of COVID19 , which suggests that they had limited knowledge about people at risk of the disease [27].

Most of respondents could clearly tell that COVID-19 disease can be prevented by practicing hand hygiene $(88.9 \%)$, social distancing $(87.2 \%)$, respiratory hygiene $(75.8 \%)$, and home confinement $(85.3 \%)$. Previous studies found similar results. For instance, in Nigeria a study revealed that prevention through hygiene (96.4\%), social distancing (93\%) and wearing a face mask (92\%) was most understood and communicated [18]. In Indonesia; the populations could list some primary prevention methods of COVID-19 such as physical distancing (78.6\%); wearing a mask $(92.5 \%)$ and washing hands (92.5\%) [19]. In India, respondents were more versed with COVID-19 preventive measures such as social distancing (91.1\%) and hand washing (96.5\%) [24]; these results could be justified by the fact that these methods are standard preventive measures used across several diseases that share similar modes of transmission.

Attitudes encompass the methods of prevention and fight against COVID-19 embedded in the lifestyle of the entire population without any distinction. According to the WHO and the $\mathrm{CDC}$, the wearing of a face mask is reserved for suspects and confirmed cases of COVID-19 [28]. In our study, 69.7\% respondents agreed that only health professionals should practice hand hygiene compared to $81.6 \%$ who argued that everyone should practice hand hygiene (washing or disinfecting hands) [29]. Also, 84.7\% of respondents agreed that everyone should wear a face mask in order to protect itself against the COVID-19 virus. This result is greater than that obtained by Xiaopen et $\mathrm{Al}$ where $65 \%$ of the participants agreed to wear a face mask [25]. Moreover, regarding the vaccine, only $16.1 \%$ of respondents agreed they would receive a potential anti-COVID-19 vaccine. This result seems close to that of Reuben et al where only $29 \%$ people accepted a vaccine against COVID-19 although about (61.8\%) declared not having enough confidence to doctors [18].

Regarding practices, the majority supported that consistently wearing a face mask provides a more efficient protective effect against COVID-19 than occasionally wearing them $(84.7 \%$ vs. $52.9 \%)$. This result is similar to that obtained in a study in India where $73 \%$ reported regularly wearing a face mask [19]. This finding could be justified by the presence of individuals from the lower socioeconomic bracket of society amongst the respondents as found by the study carried out in the South-West region of Cameroon. Only $21.7 \%$ of the respondents had bought a mask while $95 \%$ knew that the wearing of a face mask could prevent the disease [16]. Moreover, most respondents $(66 \%)$ always washed their hands as did those in India (86\%) [19] who also always use a hydro-alcoholic solution to disinfect the hands (53.1\%); always avoid touching their nose, mouth and eyes with soiled hands (55.9\%); always sneezing and coughing into the crease of the elbow or into a disposable tissue $(63.06 \%)$ and who always avoid contact greetings $(68.1 \%)$. In addition, $64.35 \%$ of our respondents said they always avoided overloaded vehicles; always refrain from visiting crowds people $61.5 \%$; always stay at home (65.4\%); always keep children at home since the closure of schools and universities (73.4\%); and 49.8\% stated that they were always influenced by those around 
them with regard to their practices. These results are similar to the study from India where $87 \%$ reported washing their hands with soap and water regularly and (73\%) reported regularly wearing a face mask [19]. These practices corroborates advertisements on social media and elsewhere since the start of the pandemic.

\section{Conclusion}

All in all, our investigation which consisted in assessing the knowledge, attitudes and practices of populations on barriers and preventives measures against COVID-19 in two cities of Cameroon, portrayed that that, most of the respondents had a good knowledge of the origin of the disease, its modes of transmission, its signs and symptoms and the people at risk and the barrier and preventive measures. Respondents' attitudes were good vis-à-vis the existing barrier measures prescribed by the government. However, few respondents with a primary level of education agreed to benefit from a possible vaccine $(\mathrm{P}=0.02)$. Practices varied from low to moderate depending on the determinants (sex, marital status, level of education, etc.). Our findings show room for sensitization geared towards encouraging the population to comply all strategies put in place to fight against the adverse effects of the pandemic. Further studies are required to assess the impact of strategies undertaken by the decision makers, to roll out the COVID-19 pandemic in Cameroon.

\section{List of Abbreviations}

ARDS: Acute Respiratory Distress Syndrome

ORa: Odds Ratio

WHO: World Health Organization

\section{Conflict of Interest}

Authors declare no competing interest

\section{Authors' Contributions}

DIC, VTN and RKW conceived the idea and designed the study. DIC, ML, AMA, and OTN scrutinized all relevant information and draft the manuscript. RKW, EL, conducted the field study. GT, EN, ANN, YVYVMM, HMM and CAO analyzed the data. All authors read and approved the final manuscript.

\section{Financial Support}

Prevention and Control Foundation.

\section{Acknowledgements}

The authors thank all the participants in the communities of Douala and Bangangté who have accepted to participate in this study.

\section{References}

[1] COVID-19 situation update worldwide, as of 16 June 2020 [Internet]. European Center for Disease Prevention and Control. [cited June 17, 2020]. Available at: https://www.ecdc.europa.eu/en/geographical-distribution2019-ncov-cases.

[2] Borges do Nascimento IJ, Cacic N, Abdulazeem HM, von Groote TC, Jayarajah U, Weerasekara I, et al. Novel Coronavirus Infection (COVID-19) in Humans: A Scoping Review and Meta-Analysis. Journal of Clinical Medicine. 2020 Apr; 9 (4): 941.

[3] Olum R, Chekwech G, Wekha G, Nassozi DR, Bongomin F. Coronavirus Disease-2019: Knowledge, Attitude, and Practices of Health Care Workers at Makerere University Teaching Hospitals, Uganda. Front Public Health [Internet]. 30 Apr 2020 [cited 17 Jun 2020]; 8. Available at: https://www.ncbi.nlm.nih.gov/pmc/articles/PMC7204940/.

[4] Knowledge, Attitude, and Practices of Healthcare Workers Regarding the Use of Face Mask to Limit the Spread of the New Coronavirus Disease (COVID-19) [Internet]. [cited June 17, 2020]. Available at: https://www.ncbi.nlm.nih.gov/pmc/articles/PMC7241223/.

[5] Zhong BL, Luo W, Li HM, Zhang QQ, Liu XG, Li WT, et al. Knowledge, attitudes, and practices towards COVID-19 among Chinese residents during the rapid rise period of the COVID-19 outbreak: a quick online cross-sectional survey. Int J Biol Sci. 2020 Mar 15; 16 (10): 1745-52.

[6] Track the spread of COVID-19 around the world | Le Devoir [Internet]. [cited 7 Nov 2020]. Available at: https://www.ledevoir.com/documents/special/20-03_covid19carte-dynamique/index.html.

[7] Gehanno JF, Bonneterre V, Andujar P, Pairon JC, Paris C, Petit A, et al. Arguments for a possible airborne transmission of SARS-CoV-2 in the COVID-19 crisis. Archives of Occupational Diseases and the Environment [Internet]. May 4, 2020 [cited July 25, 2020]; Available at: https://www.ncbi.nlm.nih.gov/pmc/articles/PMC7198203/.

[8] Harapan H, Itoh N, Yufika A, Winardi W, Keam S, Te H, et al. Coronavirus disease 2019 (COVID-19): A literature review. J Infect Public Health. May 2020; 13 (5): 667-73.

[9] Shereen MA, Khan S, Kazmi A, Bashir N, Siddique R. COVID-19 infection: Origin, transmission, and characteristics of human coronaviruses. Journal of Advanced Research. 1 Jul 2020; $24: 91-8$

[10] Guo YR, Cao QD, Hong ZS, Tan YY, Chen SD, Jin HJ, et al. The origin, transmission and clinical therapies on coronavirus disease 2019 (COVID-19) outbreak - an update on the status. Military Medical Research. 2020 Mar 13; 7 (1): 11.

[11] Li X, Xu S, Yu M, Wang K, Tao Y, Zhou Y, et al. Risk factors for severity and mortality in adult COVID-19 inpatients in Wuhan. J Allergy Clin Immunol. Jul 2020; 146 (1): 110-8.

[12] Licourt J. Coronavirus: what do the different stages of the government plan represent? [Internet]. Le Figaro.fr. 2020 [cited 7 Nov 2020]. Available at: https://www.lefigaro.fr/sciences/coronavirus-que-represententles-differents-stades-du-plan-gouvernemental-20200229. 
[13] ALdowyan N, Abdallah AS, El-Gharabawy R. Knowledge, Attitude and Practice (KAP) Study about Middle East Respiratory Syndrome Coronavirus (MERS-CoV) among Population in Saudi Arabia. int arch med [Internet]. 2017 Oct 2 [cited 2020 May 9]; 10. Available at: http://imedicalsociety.org/ojs/index.php/iam/article/view/2736.

[14] GOVERNMENT STRATEGY FOR RESPONSE TO THE CORONAVIRUS PANDEMIC (COVID-19) Services of the Prime Minister [Internet]. [cited 27 Apr 2020]. Available at: https://www.spm.gov.cm/site/?q=fr/content/strategiegouvernementale-de-riposte-face-la-pandemie-decoronavirus-COVID-19.

[15] Nicholas T, Mandaah FV, Esemu SN, Vanessa ABT, Gilchrist KTD, Vanessa LF, et al. COVID-19 knowledge, attitudes and practices in a conflict affected area of the South West Region of Cameroon. The Pan African Medical Journal [Internet]. May 13, 2020 [cited Aug 26, 2020]; 35 (34). Available at: https://www.panafrican-medjournal.com/content/series/35/2/34/full/.

[16] Abdelhafiz AS, Mohammed Z, Ibrahim ME, Ziady HH, Alorabi M, Ayyad M, et al. Knowledge, Perceptions, and Attitude of Egyptians Towards the Novel Coronavirus Disease (COVID-19). J Community Health [Internet]. 21 Apr 2020 [cited 17 Jun 2020]; Available at: http://link.springer.com/10.1007/s10900-020-00827-7.

[17] Yue S, Zhang J, Cao M, Chen B. Knowledge, Attitudes and Practices of COVID-19 Among Urban and Rural Residents in China: A Cross-sectional Study. J Community Health [Internet]. August 5, 2020 [cited August 26, 2020]; Available at: https://doi.org/10.1007/s10900-020-00877-x.

[18] Reuben RC, Danladi MMA, Saleh DA, Ejembi PE. Knowledge, Attitudes and Practices Towards COVID-19: An Epidemiological Survey in North-Central Nigeria. J Community Health. 7 Jul 2020; 1-14.

[19] Dkhar SA, Quansar R, Saleem SM, Khan SMS. Knowledge, attitude, and practices related to COVID-19 pandemic among social media users in J\&K, India. Indian Journal of Public Health. 2020 Jan 6; 64 (6): 205.

[20] Afzal MS, Khan A, Qureshi UUR, Saleem S, Saqib MAN, Shabbir RMK, et al. Community-Based Assessment of Knowledge, Attitude, Practices and Risk Factors Regarding COVID-19 Among Pakistanis Residents During a Recent Outbreak: A Cross-Sectional Survey. J Community Health. 13 Jul 2020; 1-11.

[21] Lau LL, Hung N, Go DJ, Ferma J, Choi M, Dodd W, et al. Knowledge, attitudes and practices of COVID-19 among income-poor households in the Philippines: A cross-sectional study. J Glob Health [Internet]. [cited 23 Jul 2020]; 10 (1). Available

https://www.ncbi.nlm.nih.gov/pmc/articles/PMC7294392/.

[22] Salman M, Mustafa ZU, Asif N, Zaidi HA, Hussain K, Shehzadi N, et al. Knowledge, attitude and preventive practices related to COVID-19: a cross-sectional study in two Pakistani university populations. Drugs Ther Perspect. May 9, $2020 ; 1-7$

[23] Cvetković VM, Nikolić N, Radovanović Nenadić U, Öcal A, K. Noji E, Zečević M. Preparedness and Preventive Behaviors for a Pandemic Disaster Caused by COVID-19 in Serbia. Int J Environ Res Public Health [Internet]. Jun 2020 [cited 23 Jul 2020]; 17 (11). Available at: https://www.ncbi.nlm.nih.gov/pmc/articles/PMC7313005/.

[24] Saefi M, Fauzi A, Kristiana E, Adi WC, Muchson M, Setiawan ME, et al. Survey data of COVID-19-related Knowledge, Attitude, and Practices among Indonesian Undergraduate Students. Data Brief [Internet]. June 12, 2020 [cited June 17, 2020]; Available at: https://www.ncbi.nlm.nih.gov/pmc/articles/PMC7291994/.

[25] Liu X, Zhang S. COVID-19: Face masks and human-tohuman transmission. Influenza and Other Respiratory Viruses. 2020 Jul 1; 14 (4): 472-3.

[26] Chhabra HS, Bagaraia V, Keny S, Kalidindi KKV, Mallepally A, Dhillon MS, et al. COVID-19: Current Knowledge and Best Practices for Orthopedic Surgeons. Indian J Orthop. May 18,$2020 ; 1-15$.

[27] Kebede Y, Yitayih Y, Birhanu Z, Mekonen S, Ambelu A. Knowledge, perceptions and preventive practices towards COVID-19 early in the outbreak among Jimma university medical center visitors, Southwest Ethiopia. PLoS One [Internet]. May 21, 2020 [cited June 17, 2020]; 15 (5). Available at: https://www.ncbi.nlm.nih.gov/pmc/articles/PMC7241810/.

[28] Tso RV, Cowling BJ. Importance of Face Masks for COVID19: A Call for Effective Public Education. Clin Infect Dis [Internet]. [cited 25 Aug 2020]; Available at: https://academic.oup.com/cid/advancearticle/doi/10.1093/cid/ciaa593/5866410.

[29] BOURQUIN S. Hand hygiene remains the most effective measure against infections [Internet]. Infirmiers.com. 2017 [cited 2 Feb 2020]. Available at: https://www.infirmiers.com/les-grandsdossiers/hygiene/hygiene-mains-oms-renouvellerecommandations.html. 